\title{
Deconfinement and chiral restoration within the SU(3) Polyakov-Nambu-Jona-Lasinio and entangled Polyakov-Nambu-Jona-Lasinio models in an external magnetic field
}

\author{
Márcio Ferreira, ${ }^{1}$ Pedro Costa, ${ }^{1}$ Débora P. Menezes,${ }^{2,3}$ Constança Providência,,${ }^{1}$ and Norberto N. Scoccola ${ }^{4}$ \\ ${ }^{1}$ Centro de Física Computacional, Department of Physics, \\ University of Coimbra, P-3004 - 516 Coimbra, Portugal \\ ${ }^{2}$ Departamento de Física, CFM, Universidade Federal de Santa Catarina, \\ Florianópolis, SC, CP 476, CEP 88.040-900, Brazil \\ ${ }^{3}$ Departamento de Física Aplicada, Universidad de Alicante, Ap. Correus 99, E-03080, Alicante, Spain \\ ${ }^{4}$ Department of Theoretical Physics, Comisión Nacional de Energía Atómica, 1429 Buenos Aires, Argentina; \\ CONICET, 1033 Buenos Aires, Argentina; Universidad Favaloro, 1078 Buenos Aires, Argentina.
}

(Dated: December 30, 2013)

\begin{abstract}
The behavior of the quark condensates at zero chemical potential and finite temperature subject to an external magnetic field is studied within the three flavor Nambu-Jona-Lasinio model with Polyakov loop (PNJL) and its extension, the so-called entangled PNJL model (EPNJL). A comparison with recent lattice QCD data is performed and it is shown that at $T=0 \mathrm{MeV}$ the light quark condensates are in quantitative agreement. At finite temperature, although there is an overall reasonable agreement with several lattice results, it is shown that in the lattice calculations the effect due to the electric charge quark difference is stronger and the restoration of the $u$ quark chiral symmetry starts at lower temperatures. When considering the EPNJL model with a Polyakov loop scale parameter that depends on the magnetic field, it is possible to obtain an earlier rise of the Polyakov loop with the increase of the magnetic field and due to the entanglement, the inverse magnetic catalysis is found as in the lattice QCD calculations.
\end{abstract}

PACS numbers: 24.10.Jv, 11.10.-z, 25.75.Nq / Keywords: EPNJL, PNJL, Polyakov loop,magnetic fields, transition temperatures, susceptibilities

\section{INTRODUCTION}

Understanding matter under extremely intense magnetic fields is one of the most interesting topics in modern physics due to its relevance for studies involving compact objects like magnetars [1], measurements in heavy ion collisions at very high energies [2, 3] or the first phases of the Universe [4].

The structure of the QCD phase diagram in the presence of an external magnetic field has been subject of several studies 5 [10], in particular, at zero chemical potential $\mu=0$ (the $T-e B$ plane), see [11 14] for a review. The first analysis about the influence of the magnetic field on the chiral-symmetry breaking within the framework of the standard Nambu-Jona-Lasinio (NJL) model was made in the late 1980s [15]. Recently, the influence of strong magnetic fields on the QCD phase diagram covering the whole $T-\mu$ plane was investigated within the $\mathrm{SU}(3)$ NJL in the mean field approximation [16]. For finite chemical potentials it is found that the location of the critical end point occurs at larger temperatures for stronger fields.

At zero chemical potential, almost all low-energy effective models, including the NJL-type models, as well as lattice QCD (LQCD) calculations [17 20], found an enhancement of the condensate due to the magnetic field (magnetic catalysis) independently of the temperature. The magnetic catalysis is the result of a stronger coupling of a quark-antiquark pair in the presence of the external magnetic field once the spins of the quarks are aligned along the direction of induced magnetic field according to their helicities. This effect leads to an increase of the transition temperature for chiral symmetry restoration as a function of $B$. However, a recent LQCD study [11, 21], for $N_{f}=2+1$ flavors with physical quarks and pion masses, shows a different behavior in the transition temperature, in particular, the suppression of the light condensates ( $u$ and $d$ quarks) by the magnetic field, an effect known as inverse magnetic catalysis. This suppression in the crossover region gives a nonmonotonic behavior of the condensates as a function of the magnetic field, resulting in a decreasing transition temperature with an increasing magnetic field.

In [22], the reaction of the low-lying Dirac modes to the magnetic field was studied, showing that large values of the Polyakov loop are favored by the magnetic field. Therefore, just as the chiral transition temperature, also the deconfinement transition temperature is a decreasing function of the magnetic field. A review of the predictions from low-energy approximations of QCD and previous lattice simulations is given in 11 14].

Calculations of deconfinement and chiral pseudocritical temperatures with the $\mathrm{SU}(2)$ Polyakov NJL (PNJL) 23] model and the entangled PNJL (EPNJL) 24 influenced by magnetic fields have been discussed in [13, 25]. As in almost all other low-energy QCD models, these two models predict that the critical temperature for chiral symmetry restoration increases with the increase of an external magnetic field. It was also shown that within the EPNJL that the splitting between the chiral and deconfinement transition temperatures is smaller than the splitting predicted by the PNJL model [13]. 
In the present work we analyze the effects of high intensity magnetic fields on strongly interacting matter using the SU(3) versions of the PNJL and EPNJL models, mainly through the behavior of the light quark condensate and the chiral and the deconfinement transition temperatures. Although these models do not describe the inverse magnetic catalysis effect observed in the lattice calculations of [11] at the transition temperature, some other features such as the behavior of the $u$ and $d$ condensates with an external magnetic field $B$ for zero temperature, are well reproduced. We also show that it is possible to account for the inverse magnetic catalysis effect through the parametrization of the Polyakov loop.

This paper is organized as follows. In Sec. II, we present the (E)PNJL models used in this work, the Polyakov loop potential, and the parameterizations chosen. In Sec. III, the transition temperatures are calculated as a function of the magnetic field, and results are compared with LQCD and other effective models. In Sec. IV, the behavior of the condensates with temperature and the magnetic field intensity is compared with the LQCD results. Then, in Sec. V, we show a possible way of reproducing the inverse magnetic catalysis within the EPNJL model.

\section{MODEL AND FORMALISM}

We describe quark matter subject to strong magnetic fields within the SU(3) PNJL model. The PNJL Lagrangian is given by [23]:

$$
\begin{aligned}
\mathcal{L} & =\bar{\psi}_{f}\left[i \gamma_{\mu} D^{\mu}-\hat{m}_{f}\right] \psi_{f}+\mathcal{L}_{\text {sym }}+\mathcal{L}_{\text {det }} \\
& +\mathcal{U}(\Phi, \bar{\Phi} ; T)-\frac{1}{4} F_{\mu \nu} F^{\mu \nu}
\end{aligned}
$$

where the quark sector is described by the $\mathrm{SU}(3)$ version of the NJL model which includes scalar-pseudoscalar and the t'Hooft six fermion interactions that models the axial $U(1)_{A}$ symmetry breaking [26], with $\mathcal{L}_{\text {sym }}$ and $\mathcal{L}_{\text {det }}$ given by [27]:

$$
\begin{aligned}
\mathcal{L}_{\text {sym }} & =G \sum_{a=0}^{8}\left[\left(\bar{\psi}_{f} \lambda_{a} \psi_{f}\right)^{2}+\left(\bar{\psi}_{f} i \gamma_{5} \lambda_{a} \psi_{f}\right)^{2}\right] \\
\mathcal{L}_{\text {det }} & =-K\left\{\operatorname{det}_{f}\left[\bar{\psi}_{f}\left(1+\gamma_{5}\right) \psi_{f}\right]+\operatorname{det}_{f}\left[\bar{\psi}_{f}\left(1-\gamma_{5}\right) \psi_{f}\right]\right\}
\end{aligned}
$$

where $\psi_{f}=(u, d, s)^{T}$ represents a quark field with three flavors, $\hat{m}_{c}=\operatorname{diag}_{f}\left(m_{u}, m_{d}, m_{s}\right)$ is the corresponding (current) mass matrix, $\lambda_{0}=\sqrt{2 / 3} I$ where $I$ is the unit matrix in the three flavor space, and $0<\lambda_{a} \leq 8$ denote the Gell-Mann matrices. The coupling between the magnetic field $B$ and quarks, and between the effective gluon field and quarks are implemented via the covariant derivative $D^{\mu}=\partial^{\mu}-i q_{f} A_{E M}^{\mu}-i A^{\mu}$ where $q_{f}$ represents the quark electric charge $\left(q_{d}=q_{s}=-q_{u} / 2=-e / 3\right)$, $A_{\mu}^{E M}=\delta_{\mu 2} x_{1} B$ is a static and constant magnetic field in the $z$ direction and $F_{\mu \nu}=\partial_{\mu} A_{\nu}^{E M}-\partial_{\nu} A_{\mu}^{E M}$. In the Polyakov gauge and at finite temperature the spatial components of the gluon field are neglected: $A^{\mu}=$ $\delta_{0}^{\mu} A^{0}=-i \delta_{4}^{\mu} A^{4}$. The trace of the Polyakov line defined by $\Phi=\frac{1}{N_{c}}\left\langle\left\langle\mathcal{P} \exp i \int_{0}^{\beta} d \tau A_{4}(\vec{x}, \tau)\right\rangle\right\rangle_{\beta}$ is the Polyakov loop.

To describe the pure gauge sector an effective potential $\mathcal{U}(\Phi, \bar{\Phi} ; T)$ is chosen in order to reproduce the results obtained in lattice calculations [28]:

$$
\begin{aligned}
& \frac{\mathcal{U}(\Phi, \bar{\Phi} ; T)}{T^{4}}=-\frac{a(T)}{2} \bar{\Phi} \Phi \\
& +b(T) \ln \left[1-6 \bar{\Phi} \Phi+4\left(\bar{\Phi}^{3}+\Phi^{3}\right)-3(\bar{\Phi} \Phi)^{2}\right]
\end{aligned}
$$

where $a(T)=a_{0}+a_{1}\left(\frac{T_{0}}{T}\right)+a_{2}\left(\frac{T_{0}}{T}\right)^{2}, b(T)=b_{3}\left(\frac{T_{0}}{T}\right)^{3}$. The standard choice of the parameters for the effective potential $\mathcal{U}$ is $a_{0}=3.51, a_{1}=-2.47, a_{2}=15.2$, and $b_{3}=-1.75$.

The effective potential exhibits a phase transition from color confinement $\left(T<T_{0}, \Phi=0\right)$ to color deconfinement $\left(T>T_{0}, \Phi \neq 0\right)$, where $T_{0}$ is the critical temperature for the deconfinement phase transition in pure gauge. We take $T_{0}=210 \mathrm{MeV}$ to account for quark back-reaction.

Besides the PNJL model, where $G$ denotes the coupling constant of the scalar-type four-quark interaction in the NJL sector, we consider an effective vertex depending on the Polyakov loop $(G(\Phi, \bar{\Phi}))$ : the EPNJL model. This effective vertex

$$
G(\Phi, \bar{\Phi})=G\left[1-\alpha_{1} \Phi \bar{\Phi}-\alpha_{2}\left(\Phi^{3}+\bar{\Phi}^{3}\right)\right] .
$$

generates entanglement interactions between the Polyakov loop and the chiral condensate [24]. For reasons of consistency we use $T_{0}=210 \mathrm{MeV}$ also in the EPNJL model.

The parameters of the model, $\Lambda$ a sharp cutoff in 3momentum space, only for the divergent ultraviolet integrals, the coupling constants $G$ and $K$ and the current quark masses $m_{u}^{0}$ and $m_{s}^{0}$ are determined by fitting $f_{\pi}$, $m_{\pi}, m_{K}$ and $m_{\eta^{\prime}}$ to their empirical values. We consider $\Lambda=602.3 \mathrm{MeV}, m_{u}=m_{d}=5.5 \mathrm{MeV}, m_{s}=140.7$ $\mathrm{MeV}, G \Lambda^{2}=1.385$ and $K \Lambda^{5}=12.36$ as in [29]. The parameter set $\left(\alpha_{1}, \alpha_{2}\right)$ must satisfy the triangle region $\left\{-1.5 \alpha_{1}+0.3<\alpha_{2}<-0.86 \alpha_{1}+0.32 \alpha_{2}, \alpha_{2}>0\right\}$, with $T_{0}=150 \mathrm{MeV}$. We choose $\alpha_{1}=0.25$ and $\alpha_{2}=0.10$.

The thermodynamical potential for the three flavor quark sector, $\Omega$, is written as

$$
\begin{aligned}
\Omega(T, B) & =\mathcal{U}(\Phi, \bar{\Phi}, T)+G(\Phi, \bar{\Phi}) \sum_{f=u, d, s}\left\langle\bar{q}_{f} q_{f}\right\rangle^{2} \\
& +4 K\left\langle\bar{q}_{u} q_{u}\right\rangle\left\langle\bar{q}_{d} q_{d}\right\rangle\left\langle\bar{q}_{s} q_{s}\right\rangle \\
& +\sum_{f=u, d, s}\left(\Omega_{f}^{v a c}+\Omega_{f}^{m a g}+\Omega_{f}^{m e d}\right)
\end{aligned}
$$

where the vacuum $\Omega_{f}^{v a c}$, the magnetic $\Omega_{f}^{\text {mag }}$, the medium contributions $\Omega_{f}^{\text {med }}$ and the quark condensates $\left\langle\bar{q}_{f} q_{f}\right\rangle$ have been evaluated with great detail in [30, 31]. 


\begin{tabular}{|c|c|c|c|c|c|c|c|c|}
\hline \multirow{2}{*}{$\begin{array}{c}e B \\
\left(\mathrm{GeV}^{2}\right)\end{array}$} & \multicolumn{4}{|c|}{$\begin{array}{l}\text { PNJL } \\
\end{array}$} & \multicolumn{4}{|c|}{ EPNJL } \\
\hline & $T_{c}^{u}$ & $T_{c}^{d}$ & $T_{c}^{\chi}$ & $T_{c}^{\Phi}$ & $T_{c}^{u}$ & $T_{c}^{d}$ & $T_{c}^{\chi}$ & $T_{c}^{\Phi}$ \\
\hline 0 & 200 & 200 & 200 & 171 & 187 & 187 & 187 & 184 \\
\hline 0.2 & 209 & 208 & 208 & 172 & 193 & 193 & 193 & 187 \\
\hline 0.4 & 226 & 224 & 225 & 174 & 206 & 205 & 206 & 195 \\
\hline 0.6 & 246 & 242 & 244 & 178 & 222 & 221 & 222 & 204 \\
\hline 0.8 & 267 & 257 & 262 & 182 & 240 & 237 & 238 & 214 \\
\hline 1 & 288 & 271 & 279 & 186 & 257 & 252 & 255 & 224 \\
\hline
\end{tabular}

TABLE I. Pseudo-critical temperatures in $\mathrm{MeV}$ for the chiral transition $\left(T_{c}^{\chi}=\left(T_{u}^{\chi}+T_{d}^{\chi}\right) / 2\right)$ and for the deconfinement $\left(T_{c}^{\Phi}\right)$ for both, PNJL and EPNJL, models with $T_{0}=210 \mathrm{MeV}$.

To obtain the mean field equations we must minimize the thermodynamical potential (4) with respect to $\left\langle\bar{q}_{f} q_{f}\right\rangle, \Phi$ and $\bar{\Phi}$ [30 32]. Finally, according to [21] we define the change of the light condensate due to the magnetic field as

$$
\Delta \Sigma_{f}(B, T)=\Sigma_{f}(B, T)-\Sigma_{f}(0, T)
$$

with

$$
\Sigma_{f}(B, T)=\frac{2 m_{f}}{m_{\pi}^{2} f_{\pi}^{2}}\left[\left\langle\bar{q}_{f} q_{f}\right\rangle(B, T)-\left\langle\bar{q}_{f} q_{f}\right\rangle(0,0)\right]+1
$$

where the factor $m_{\pi}^{2} f_{\pi}^{2}$ in the denominator contains the pion mass in the vacuum $\left(m_{\pi}=135 \mathrm{MeV}\right)$ and (the chiral limit of the) pion decay constant $\left(f_{\pi}=87.9\right) \mathrm{MeV}$ in PNJL model.

\section{PNJL AND EPNJL MODELS IN AN EXTERNAL MAGNETIC FIELD}

At zero temperature the chiral symmetry of QCD is explicitly broken. Consequently, at high temperature it is expected that chiral symmetry be restored. At $e B=0$ both, PNJL and EPNJL, models show a crossover transition: we can only establish a pseudo-critical temperature which depends on the observable used to define it 32. To identify the pseudo-critical temperature for the chiral transition $T_{c}^{\chi}=\left(T_{u}^{\chi}+T_{d}^{\chi}\right) / 2$ (being $T_{u}^{\chi}$ and $T_{d}^{\chi}$ the transition temperatures for $u$ and $d$ quarks, respectively) and for the deconfinement $\left(T_{c}^{\Phi}\right)$, we use the location of the peaks for the vacuum normalized quark condensates and the Polyakov loop field $\Phi$ susceptibilities given, respectively, by

$$
\begin{gathered}
C_{f}=-m_{\pi} \partial \sigma_{f} / \partial T, \sigma_{f}=\left\langle\overline{q_{f}} q_{f}\right\rangle(B, T) /\left\langle\overline{q_{f}} q_{f}\right\rangle(B, 0), \\
C_{\Phi}=m_{\pi} \partial \Phi / \partial T
\end{gathered}
$$

The multiplication by $m_{\pi}$ is only to ensure that the susceptibilities are dimensionless.

The pseudo-critical temperatures for $u$ and $d$ quark transitions become different as $e B$ increases, within both

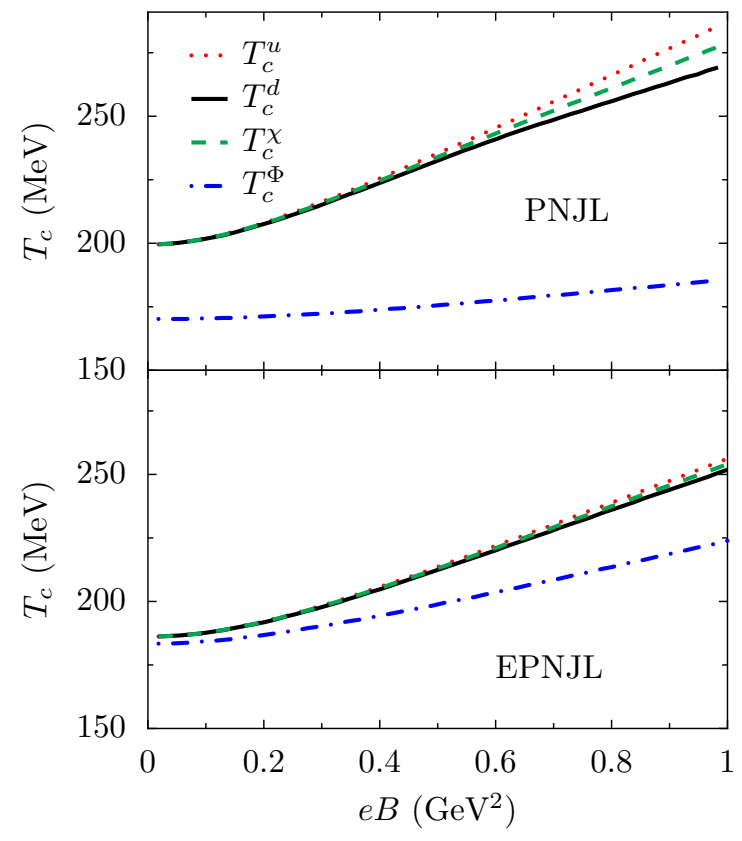

FIG. 1. Pseudo-critical temperatures for the chiral transition, $T_{u}^{\chi}, T_{d}^{\chi},\left(T_{c}^{\chi}=\left(T_{u}^{\chi}+T_{d}^{\chi}\right) / 2\right)$ and for the deconfinement $\left(T_{c}^{\Phi}\right)$ vs the magnetic field intensity for PNJL (top) and EPNJL (bottom).

PNJL and EPNJL, see Fig. 1] and Table \, although a stronger difference occurs for PNJL. Due to its larger electric charge, the $u$ quark has an effective mass that becomes larger, which is manifested in the behavior of the respective condensate, see Figs. 2 (a) and (c) respectively for PNJL and EPNJL, so the partial restoration of chiral symmetry in the $u$ sector is delayed and the respective transition occurs at a higher temperature than the transition in the $d$ sector. It is also observed that as the magnetic field becomes stronger, the separation between the temperatures $T_{c}^{\chi}$ and $T_{c}^{\Phi}$ increases, see Figs. 2 (b) and (d), and in Table \. This effect is much stronger for the PNJL than the EPNJL, see Fig. 1, In fact, although the entanglement imposed between the quarks and the Polyakov loop in the EPNJL makes both temperatures $T_{c}^{\chi}$ and $T_{c}^{\Phi}$ almost coincident if $e B=0$, a very strong magnetic field destroys this coincidence.

It is also interesting to note from Fig. 1 that for both models, we can find a phase in which quark matter is (statistically) deconfined, but chiral symmetry is still broken. As pointed out in [33] this phase can be called constituent quark phase (CQP).

In the PNJL, the magnetic field has a smaller impact on the location of the deconfinement crossover as already noticed in 25] for the $\mathrm{SU}(2)$ sector: $T_{c}^{\Phi}$ has just a weak increase, $\sim 15 \mathrm{MeV}$ if $e B$ increases from 0 to $1 \mathrm{GeV}^{2}$.

This value is comparable with the corresponding 79 $\mathrm{MeV}$ increase of the chiral transition temperature. Moreover, the Polyakov loop susceptibilities become narrower with an increasing magnetic field and eventually for sufficient strong magnetic fields a first order phase transition 

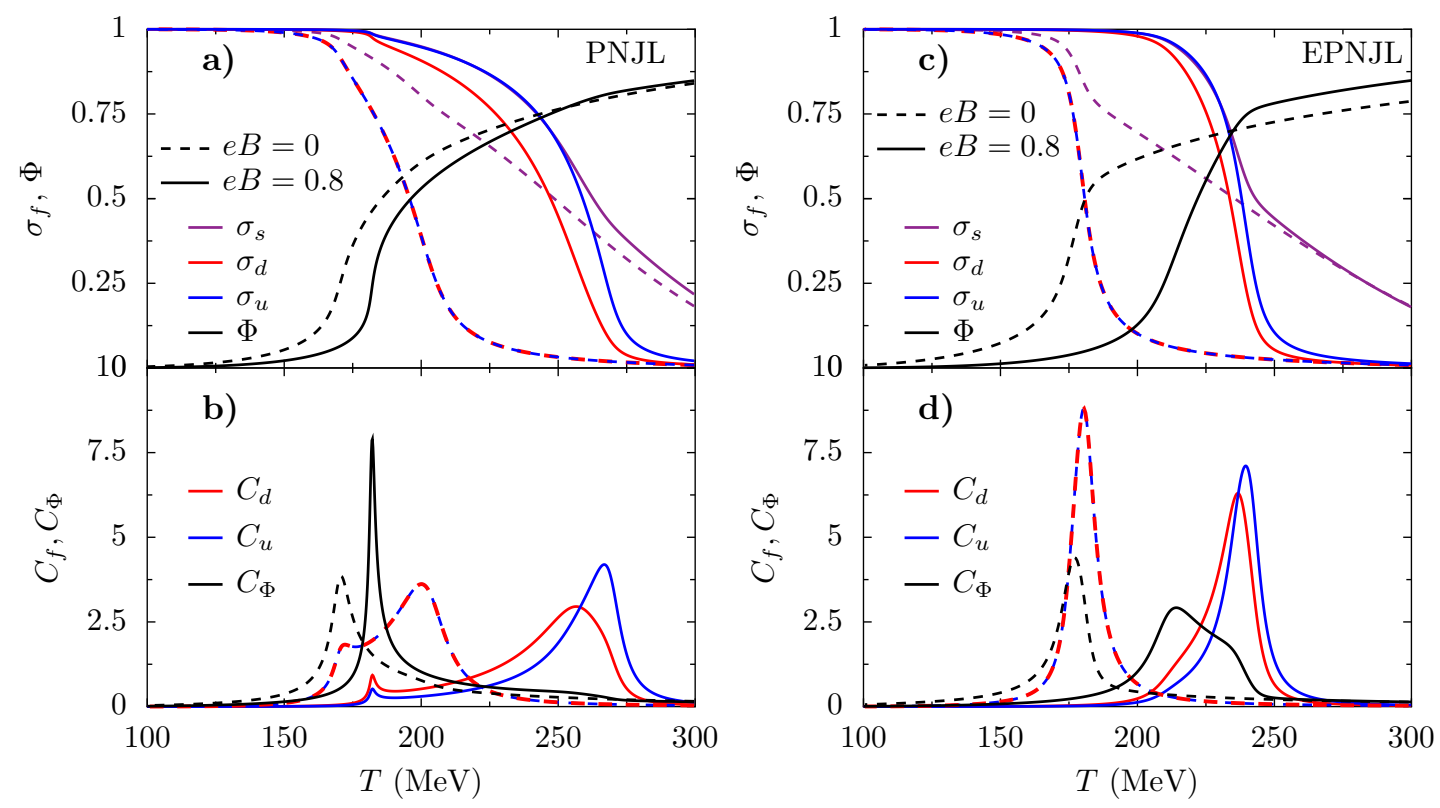

FIG. 2. Vacuum normalized condensates $\sigma_{f}$ and the Polyakov loop $\Phi$ (top) and their susceptibilities, $C_{f}$ and $C_{\Phi}$ (bottom), vs $T$ for $e B=0$ and $e B=0.8 \mathrm{GeV}^{2}$, obtained within the PNJL (left) and the EPNJL (right).

\begin{tabular}{|c|c|c|c|c|}
\hline \multirow{2}{*}{} & \multicolumn{2}{|c|}{ PNJL } & \multicolumn{2}{c|}{ EPNJL } \\
\cline { 2 - 5 } & $\mathrm{A}$ & $\alpha$ & $\mathrm{A}$ & $\alpha$ \\
\hline \hline$T_{c}^{u}(B) / T_{c}^{u}(0)$ & $1.38 \times 10^{-3}$ & 1.50 & $6.71 \times 10^{-4}$ & 1.65 \\
\hline$T_{c}^{d}(B) / T_{c}^{d}(0)$ & $1.20 \times 10^{-3}$ & 1.52 & $5.90 \times 10^{-4}$ & 1.68 \\
\hline$T_{c}^{\chi}(B) / T_{c}^{\chi}(0)$ & $1.29 \times 10^{-3}$ & 1.51 & $6.31 \times 10^{-4}$ & 1.67 \\
\hline$T_{c}^{\Phi}(B) / T_{c}^{\Phi}(0)$ & $5.87 \times 10^{-5}$ & 1.90 & $4.42 \times 10^{-4}$ & 1.61 \\
\hline
\end{tabular}

TABLE II. Coefficient $A$ and exponent $\alpha$ of the expansion of the transition temperatures for small values of the magnetic field $e B$, see Eq. (8).

takes place. It is interesting to see a quite different behavior within the EPNJL, where the deconfinement crossover suffers a shift of $40 \mathrm{MeV}$, if $e B$ increases from 0 to 1 $\mathrm{GeV}^{2}$. Due to the entanglement the Polyakov loop susceptibility peak is shifted towards higher temperatures, together with the $C_{u}$ and $C_{d}$ peaks, which move $\sim 68$ $\mathrm{MeV}$, when $e B$ goes from 0 to $1 \mathrm{GeV}^{2}$. However, also due to the entanglement interaction, the $C_{u}$ and $C_{d}$ peaks do not move to so high temperatures as in the PNJL model.

The PNJL condensate susceptibilities display small peaks around the peak of $C_{\Phi}$ related to the fastening of the phase transition induced by the Polyakov loop [32]. They do not signal a phase transition since the variation of the order parameter around this temperature is small. A similar effect is seen in the EPNJL Polyakov loop susceptibility close to the peak of the $C_{u}$ and $C_{d}$.

To try to understand the dependence of $T_{c}^{i}$ on $e B$ we perform the parametrization of the phase transition line introduced in Refs. 17, 34], valid for small values of the magnetic field $\left(e B \lesssim 0.5 \mathrm{GeV}^{2}\right)$ :

$$
\frac{T_{c}^{i}(B)}{T_{c}^{i}(0)}=1+A\left(\frac{e B}{m_{\pi}^{2}}\right)^{\alpha}
$$

The numerical values of the best-fit coefficients are given in Table \. The results show what Fig. 1 also reveals: the curvature for the Polyakov transition is softer in the PNJL model than in the EPNJL model due to the entanglement interactions between the Polyakov loop and the chiral condensate in this last model.

\section{THE PNJL AND EPNJL MODELS VERSUS THE LATTICE RESULTS}

Next, we focus our study on the quark condensates as functions of $e B$ at $T=0$, having in mind the comparison of the PNJL and EPNJL models with lattice results for the quark condensates subject to an external magnetic field 21]. Note that for $T=0$ the three models NJL, PNJL, and EPNJL coincide. In Fig. 3 (a) we compare the PNJL model results for the change of the renormalized condensate $\overline{\Delta \Sigma}=\Delta\left(\Sigma_{u}+\Sigma_{d}\right) / 2$ with lattice results extracted from 21]. Our results agree quantitatively well and even at $e B=1 \mathrm{GeV}^{2}$, the discrepancy of the order of $\sim 10 \%$, is much smaller than the prediction of chiral perturbation theory and SU(2) PNJL model (see Ref. [21]). As expected, for small fields $\left(e B<m_{\pi}^{2}\right)$ we obtain a quadratic dependence of $\overline{\Delta \Sigma}$ on $e B$ and a linear dependence for higher fields $\left(e B \gg m_{\pi}^{2}\right)$ [18]. In Table III we present the lattice results for the light condensates at zero temperature, as functions of $e B$ 21], together with the results obtained for the PNJL model. The average of the 


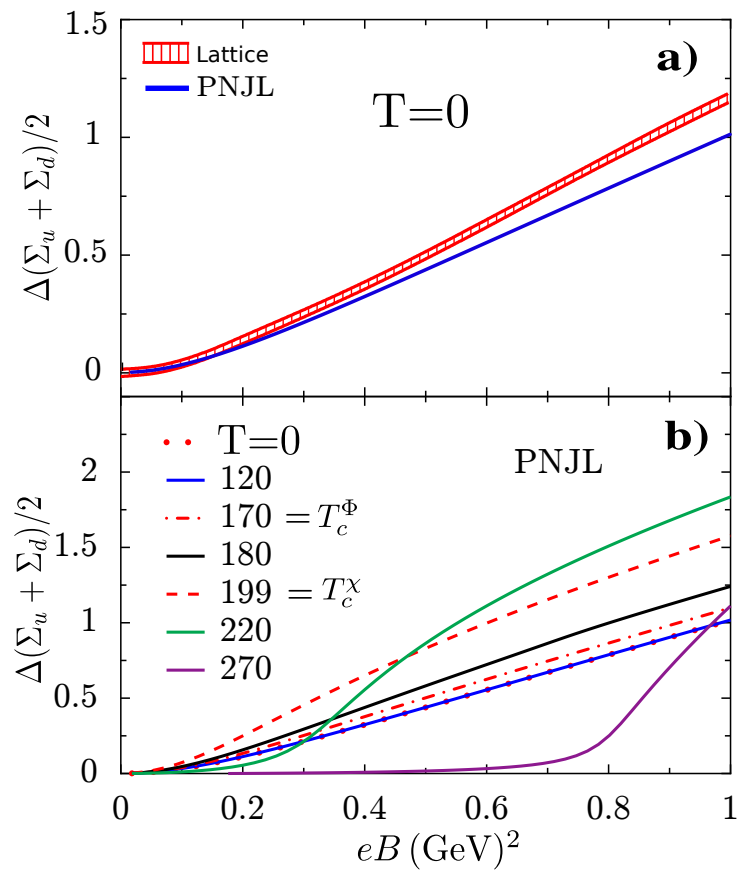

FIG. 3. $\overline{\Delta \Sigma}$ vs $e B$ (a) at $T=0$ for PNJL and the lattice results of [21] and (b) within PNJL for several temperatures (in $\mathrm{MeV}$ ) close to the transition temperature.

\begin{tabular}{|c|c|c|c|c|c|c|}
\hline \multirow{2}{*}{$T=0$} & \multicolumn{2}{|c|}{$e B=0$} & \multicolumn{2}{|c|}{$e B=0.2 \mathrm{GeV}^{2}$} & \multicolumn{2}{|c|}{$e B=0.4 \mathrm{GeV}^{2}$} \\
\hline & $+/ 2$ & - & $+/ 2$ & - & $+/ 2$ & - \\
\hline (E)PNJL & 1 & 0 & 1.11 & 0.08 & 1.32 & 0.23 \\
\hline Latt. [21] & 1 & 0 & $1.14(2)$ & $0.09(2)$ & $1.37(2)$ & $0.28(2)$ \\
\hline \multirow{2}{*}{$T=0$} & \multicolumn{2}{|c|}{$e B=0.6 \mathrm{GeV}^{2}$} & \multicolumn{2}{|c|}{$e B=0.8 \mathrm{GeV}^{2}$} & \multicolumn{2}{|c|}{$\mid e B=1.0 \mathrm{GeV}^{2}$} \\
\hline & $+/ 2$ & - & $+/ 2$ & - & $+/ 2$ & - \\
\hline (E)PNJL & 1.55 & 0.40 & 1.79 & 0.58 & 2.02 & 0.76 \\
\hline Latt. [21] & $1.63(3)$ & $0.47(3)$ & $\mid 1.90(3)$ & $0.67(3)$ & |2.16(3) & $0.87(3)$ \\
\hline
\end{tabular}

TABLE III. Results obtained for the PNJL (EPNJL) model together with the continuum extrapolated lattice results for the light condensates at $T=0$ [21]. Columns labeled " $+/ 2$ " contain the light condensates average, while those with "-" contain the difference.

light condensates ("+/2") is in very good agreement with lattice results, especially at low magnetic fields. Even for $e B=1 \mathrm{GeV}^{2}$ the average of the light condensates does not differ more than $\sim 10 \%$.

In Fig. 3 (b) the average of $u$ and $d$ condensates is plotted as a function of the magnetic field intensity for several temperatures in the PNJL model. For $T<T_{c}^{\chi}(e B=0)$ the condensates average increases with $e B$ due to the magnetic catalysis effect, being its value greater the higher the temperature. When $T>T_{c}^{\chi}(e B=0)$ we are in the region where the partial restoration of chiral symmetry already took place. In this region there are two competitive effects: the partial restoration of chiral symmetry and the magnetic catalysis. The former effect

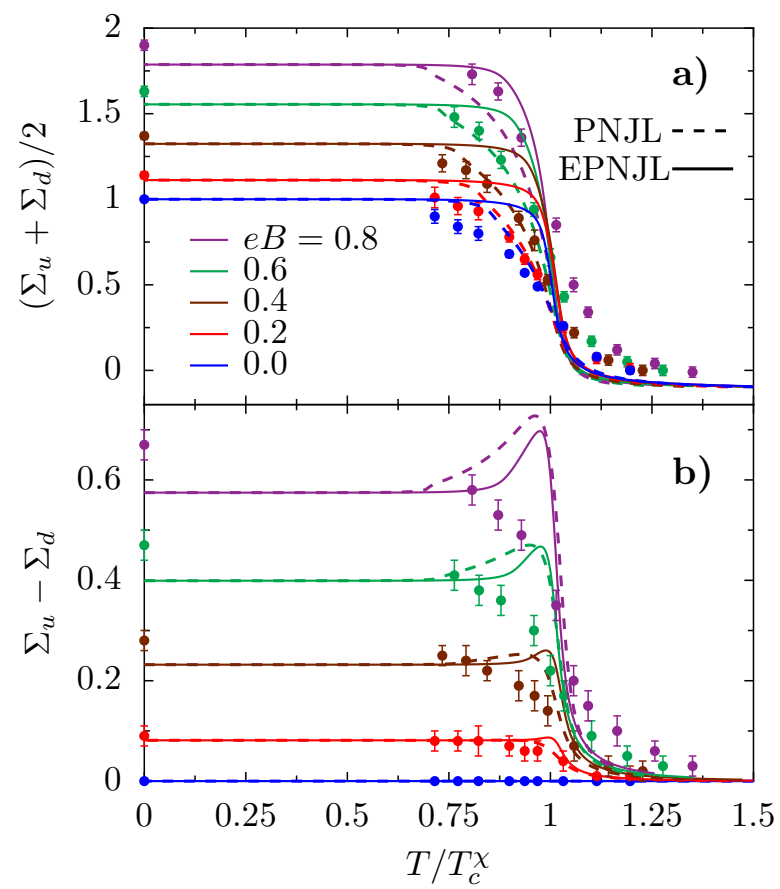

FIG. 4. (a) Light quark condensate average and (b) light quark condensate difference and corresponding lattice results taken from [11, 21] vs $T$ for several values of $e B$.

prevails at lower values of $e B$, making the condensates average approximately zero. The latter effect becomes dominant as the magnetic field increases and the average condensate becomes nonzero. Let us take as an example the case $T=270 \mathrm{MeV}$ : since $T=270 \mathrm{MeV}$ is larger than $T_{c}^{\chi}(e B=0) \mathrm{MeV}$, the average condensate is approximately zero for small values of $e B$ and starts to increase around $e B=0.6 \mathrm{GeV}^{2}$, a magnetic field strong enough to prevent the restoration of chiral symmetry that would have occurred at zero magnetic field.

The results within the EPNJL model are qualitatively similar to the results of the PNJL model. However, it is important to make some comments on the new features of EPNJL. From Table \it is seen that the coincidence existing between the deconfinement and chiral transition temperatures at $e B=0$ is destroyed in the presence of an external magnetic field. When compared with PNJL, the effect of entanglement present in the EPNJL is seen on the larger (smaller) increase of $T_{c}^{\Phi}\left(T_{c}^{\chi}\right)$ as already explained.

In Table III we also list the values for the difference between $u$ and $d$ quark condensates ("-"), at $T=0$, in comparison with lattice calculations. Once again the results are in good agreement namely for lower values of $e B$, although, a significative difference between PNJL and lattice calculations occurs for larger values of $e B$, with the lattice predicting a larger difference between both condensates. This means that the effect due to the electric charge quark difference is stronger in lattice calculations. 

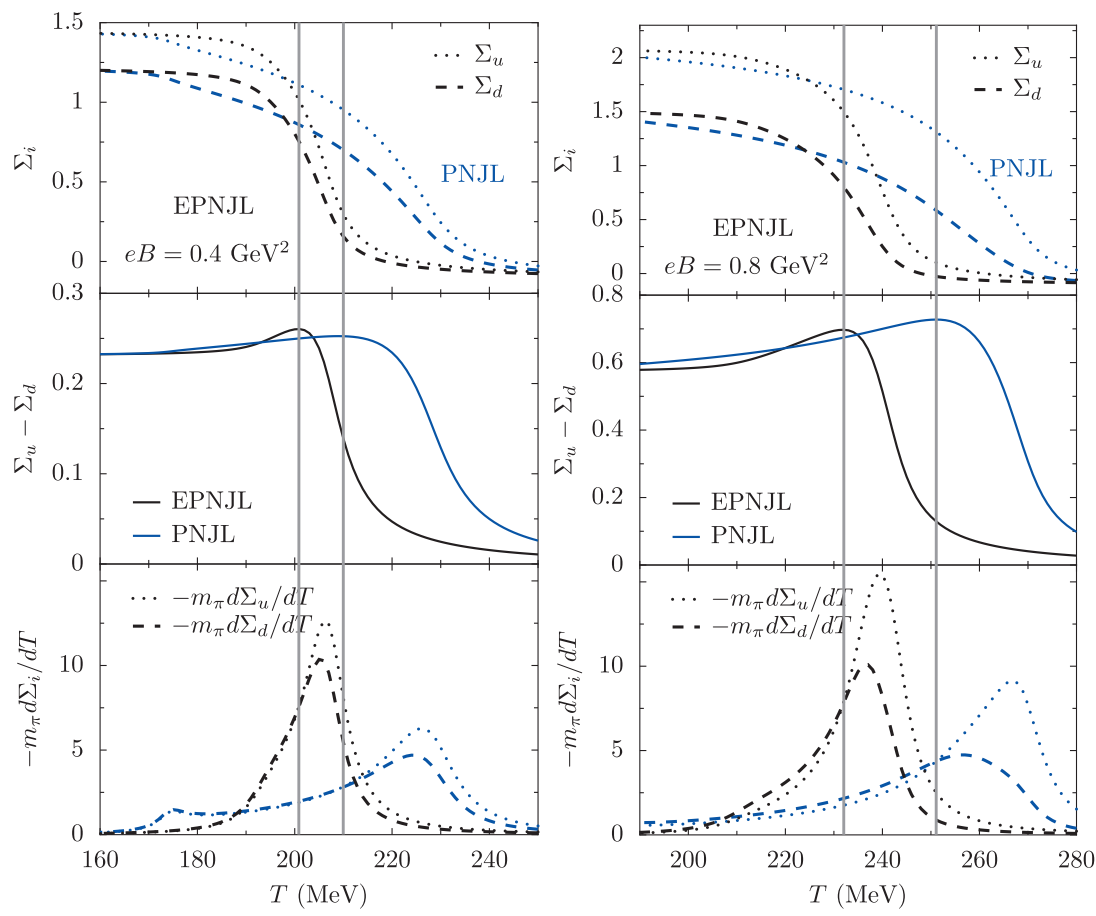

FIG. 5. The quark condensates $\Sigma_{i}$ (top), the difference between the $u$ and $d$ quark condensates (middle), and derivatives of the quark condensates versus $T$ for PNJL (black) and EPNJL (red) and two magnetic field intensities, $e B=0.4 \mathrm{GeV}^{2}$ (top figures) and $e B=0.8 \mathrm{GeV}^{2}$ (bottom figures).

In Fig. 团 (a) the average and (b) the difference between light quark condensate is plotted as a function of $T / T_{c}^{\chi}(e B)$ for several values of $e B$ for PNJL (dashed lines), EPNJL (full lines) and lattice data [11, 21]. The temperature normalization was done in order to remove the inverse magnetic catalysis effect in the lattice results. The lattice results for the condensates and the critical temperatures were taken from [11, 21]. The comparison plotted in Fig. 4 (a) for the average of the light condensates shows that in general PNJL and EPNJL have the same behavior as the lattice results except for a too fast drop at the respective transition temperatures. The effect of a stronger magnetic catalysis for the $u$ quark, due to its larger electric charge, present in both models at finite temperatures is clear in Fig. 4 (b): the larger the magnetic field the larger the difference between $u$ and $d$ condensates, and the respective chiral transition temperatures (see Table \). This feature is particularly strong close to the transition temperature, where the curves for stronger fields have a larger bump. This behavior was already found in 35] where the authors have employed the instanton-liquid model, modified by the HarringtonShepard caloron solution at finite temperature to investigate the chiral restoration in the presence of a strong external magnetic field. After the transition temperature $T_{c}^{\chi}$, the masses of the quarks are smaller, due to the partial restoration of chiral symmetry, prevailing this effect over the magnetic catalysis. For these temperatures the $u$ and $d$ quark condensate difference is small.

The bump appears in the $u, d$ condensate difference both within PNJL and EPNJL and becomes stronger as the magnetic field increases (see Fig. 4). To understand the reason of this feature, we show in Fig. 5 the condensates $\Sigma_{i}, \Sigma_{u}-\Sigma_{d}$, and the $\Sigma_{i}$ susceptibilities, for $e B=0.4$ and $e B=0.8 \mathrm{GeV}^{2}$ in both models, removing the temperature renormalization. The appearance of the peaks is due to the change of the behavior of the susceptibilities. This effect is clearer for $e B=0.8 \mathrm{GeV}^{2}$. The vertical gray lines indicate the temperature of the $\Sigma_{u}-\Sigma_{d}$ maximum. For temperatures below this value, $\left|d \Sigma_{d} / d T\right|>\left|d \Sigma_{u} / d T\right|$, and above the bump the opposite happens. Due to the charge difference, the magnetic catalysis is stronger for $u$ than $d$ quarks, therefore (a) at lower temperatures, the decrease of the $d$ condensate with temperature is faster, because the partial restoration of chiral symmetry in the $u$ sector is delayed; (b) at temperatures close to the transition temperature, $\Sigma_{u}$ must decrease with temperature faster than the $\Sigma_{d}$. Therefore, $\Sigma_{u}-\Sigma_{d}$ remains constant at low temperatures, then, it increases up to a value below the $d$ chiral transition temperature, and finally, decreases until the chiral symmetry is restored. At variance, the lattice results [21], predict a monotonous decrease of $\Sigma_{u}-\Sigma_{d}$ with $T$, possibly showing that the partial restoration of chiral symmetry in both the $u$ and $d$ sector occur simultaneously.

Lattice results [11] show that the transition remains an analytic crossover for magnetic fields at least up to $1 \mathrm{GeV}^{2}$. In particular, the $u$ quark transition width decreases only mildly and the height grows significantly. In Fig. 6] the susceptibilities $C_{u}$ and $C_{d}$ are plotted as a 


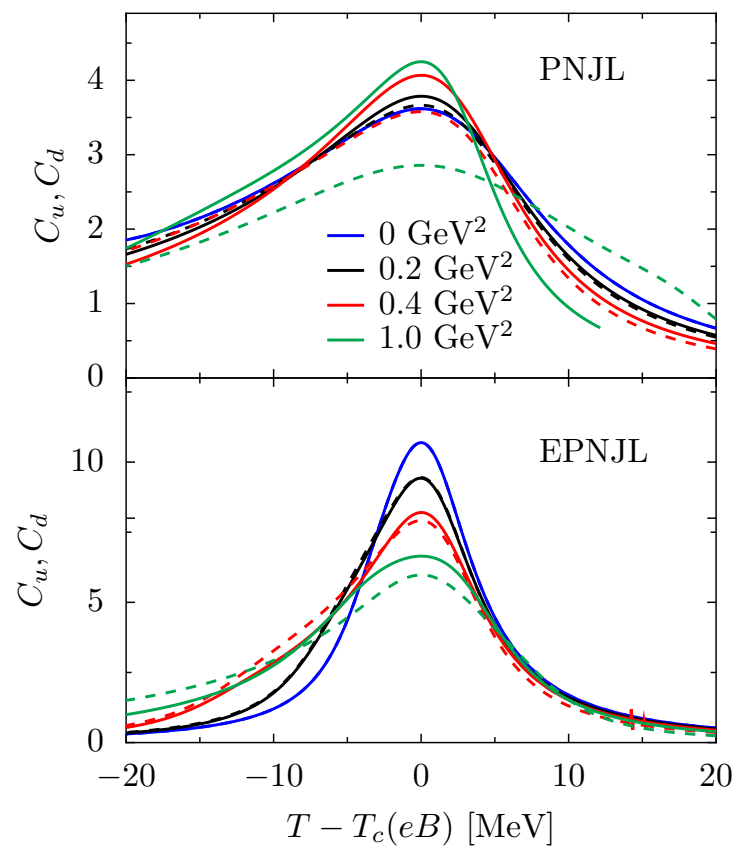

FIG. 6. Relative changes in the temperature dependence of chiral susceptibility for quark $u$ (full lines) and $d$ (dashed lines) and for different values of $e B$, within PNJL (top) and EPNJL (bottom). The width increases with $e B$.

function of $T_{c}-T_{c}(e B)$ for several magnetic field intensities. In both models the widths of these susceptibilities do not change much with the magnetic field intensity even if they show opposite behaviors, which can be seen by taking the width of the peak at half maximum. In the PNJL model for the $u$ quark we see that there is a decrease from $\sim 31.1$ to $\sim 21.7 \mathrm{MeV}$ as the external field is increased from zero to $e B=0.4 \mathrm{GeV}^{2}$ and then it remains almost constant. The $e B=0$ width of the peak at half maximum of $C_{u}$ and $C_{d}$ is large due to the influence of the deconfinement transition which can be seen in Fig. 2 (b) (red and blue dashed lines). For the $d$ quark we have a similar behavior except for $e B \approx 1$ $\mathrm{GeV}^{2}$ where there is a strong increase of the width due to the influence of the $u$ quark transition on the $d$ quark transition. The influence of the $d$ quark transition on the $u$ quark transition also exists but it is very weak. Both behaviors are due to the split of the respective transitions as $e B$ increases.

Contrary to what happens in the PNJL model, the behavior for the EPNJL model shows an increase of the widths from $\sim 8.5(8.5)$ to $\sim 14.7(16.7) \mathrm{MeV}$ as the external field increases from zero to $e B=1 \mathrm{GeV}^{2}$ for the $u(d)$ quarks. This behavior is mainly explained by the separation of the deconfinement and chiral transitions and the influence of the first one on the second one due to the entanglement interaction. The height also presents different behaviors in both models, and different from LQCD: in the PNJL the height increases $\sim 18 \%$ for $C_{u}$ and decreases $\sim 21 \%$ for $C_{d}$, for $e B$ between 0 and 1
$\mathrm{GeV}^{2}$. Within the EPNJL, $C_{u}$ decreases $\sim 38 \%$ from $e B=0$ to $1 \mathrm{GeV}^{2}$. This result is a consequence of the entanglement with the Polyakov loop (see Fig. 22).

Due to the charge difference, the magnetic field softens more the $d$ transition than the $u$ transition once the peaks height is always bigger for the $u$ quark in both models.

\section{INVERSE MAGNETIC CATALYSIS AT FINITE $T$}

So far, we have seen that SU(3) (E)PNJL reproduces quite well the lattice QCD quark condensate behavior in an external magnetic field except for the inverse magnetic catalysis effect predicted by lattice QCD calculations at temperatures of the order of the transition temperature and high magnetic fields, and the width of the susceptibilities at the transition. In the (E)PNJL the deconfinement is described by the Polyakov loop which couples weakly to the magnetic field as referred above. It should be noted that the Polyakov loop potential was originally parametrized in order to reproduce the pure gluonic lattice data.

Later, it was realized that the inclusion of dynamical quarks leads to a decrease of the scale parameter $T_{0}$. Since strong magnetic fields have certainly an effect on dynamical quarks, one expects that their presence could affect the value of $T_{0}$. In a recent lattice calculation [22], it is argued that the inverse magnetic catalysis may be a consequence of how the gluonic sector reacts to the presence of a magnetic field: the distribution of gluon fields may change as an indirect effect of the magnetic field mediated by quark loops that destroys the chiral condensate. This behavior happens around and above the deconfinement temperature differently from lattice results at zero temperature [18], where the modified distribution of gluon fields contributes to increase the magnetic catalysis. The authors have shown that the change of the renormalized Polyakov loop increases sharply with the magnetic field around the transition temperature, and that the transition temperature decreases with the magnetic field. Therefore, the back-reaction of the quarks on the gauge fields should be incorporated in effective models in order to describe the inverse magnetic catalysis.

On the other hand, it is known the effect of screening of the gluon interactions in a magnetic field in the region of momenta relevant for the chiral symmetry breaking dynamics [36]. In this region, gluons acquire a mass $M_{g}$ of order $\sqrt{N_{f} \alpha_{s}|e B|}$, with $M_{g}$ being the mass of a quark-antiquark composite state coupled to the gluon field. In a strong enough magnetic field, this mass $M_{g}$ for gluons becomes larger. This, along with the property of the asymptotic freedom $\left(\alpha_{s}\right.$ decreases with increasing $e B)$, leads to the suppression of the chiral condensate. It was also shown that the confinement scale in the presence of a strong magnetic field is much less than the corresponding scale in QCD without magnetic field [36]. Also calculations in the large $N_{c}$ limit of the quark mass 
gap in a magnetic field showed that the quark mass gap does not grow much beyond $\Lambda_{Q C D}$. In this scenario, the screening effects for gluons were not explicitly taken into account. By introducing $1 / N_{c}$ corrections the screening effects should grow as $e B$ increases leading to the reduction of the pseudo-critical temperatures [37].

One possible approach to mimic the reaction of the gluon sector to the presence of an external magnetic field is to choose a magnetic field dependent $T_{0}(e B)$, in order to reproduce the correct transition temperatures given by lattice [11, 22]. This type of procedure on $T_{0}$ had already been proposed in [38] in a different context: based on renormalization group arguments, an explicit quark chemical potential and $N_{f}$ dependence on $T_{0}$ in the Polyakov loop potential takes into account the backreaction of the quark degrees of freedom on the Polyakov loop.

We next start from the lattice results and analyze whether they can be reproduced within the PNJL and/or EPNJL models imposing a dependence of the Polyakov loop on the magnetic field. This dependence will be included through the parameter $T_{0}$. However, it should be pointed out that a too small value of $T_{0}$ leads to a first order phase transition within PNJL and EPNJL, and, therefore, the range of $T_{0}$ values of interest is limited to the values that maintain the crossover transition.

Within the PNJL it is not possible to implement the above scheme because the chiral transition temperatures increase strongly with the external magnetic field. In order to bring these temperatures down it would be necessary to use very small values of $T_{0}$, for which the deconfinement phase transition becomes of first order. However, within EPNJL the chiral condensates and the Polyakov loop are entangled. Thus, the chiral transition temperatures are pulled down to temperatures close to the deconfinement transition temperature. This model, however, still predicts a first order transition for both transitions when $T_{0}$ is too small at moderate magnetic fields.

In order to proceed, we take a magnetic field dependent $T_{0}(e B)$ of the form

$$
T_{0}(e B)=T_{0}(e B=0)+\zeta(e B)^{2}+\xi(e B)^{4},
$$

fitted to the transition temperature for the strange quark number susceptibility data, that is viewed as a quantity signaling the deconfinement transition, extracted from [11].

For $e B=0$ we have $T_{0}=186 \mathrm{MeV}$, in agreement with the $T_{0}$ value that encodes the back-reaction of the matter sector to the gluon sector for $N_{f}=2+1$ massless flavors [39]. The respective transition temperatures are $T_{c}^{\Phi}=173.9$ and $T_{c}^{\chi}=176.0 \mathrm{MeV}$ (see Table [V). The values of $\zeta$ and $\xi$ in Eq. (9) are also given in Table IV. This parametrization of $T_{0}(e B)$ can lead to inverse magnetic catalysis, see Fig. 7 blue line, and allows to describe the back-reaction on the Polyakov loop due to the presence of an external magnetic field for $e B \lesssim 0.25$ $\mathrm{GeV}^{2}$. Above this value a first order phase transition

\begin{tabular}{cccccc}
\hline $\begin{array}{c}T_{0}(e B=0) \\
{[\mathrm{MeV}]}\end{array}$ & $\begin{array}{c}T_{c}^{\Phi} \\
{[\mathrm{MeV}]}\end{array}$ & $\begin{array}{c}T_{c}^{\chi} \\
{[\mathrm{MeV}]}\end{array}$ & $e B^{\text {max }}$ & $\begin{array}{c}\zeta \\
{\left[\mathrm{GeV}^{2}\right]}\end{array}$ & $\begin{array}{c}\xi \\
{\left[\mathrm{MeV} / \mathrm{GeV}^{4}\right]}\end{array}$ \\
\hline 186 & 173.9 & 176.0 & 0.25 & -646.491 & 78.8961 \\
195 & 177.4 & 179.9 & 0.3 & -845.467 & 2813.38 \\
270 & 214.0 & 216.0 & 0.61 & -162.632 & -545.027 \\
\hline
\end{tabular}

TABLE IV. Pseudo-critical temperatures for chiral transition and for the deconfinement in the EPNJL model for different values of $T_{0}(e B=0)$.

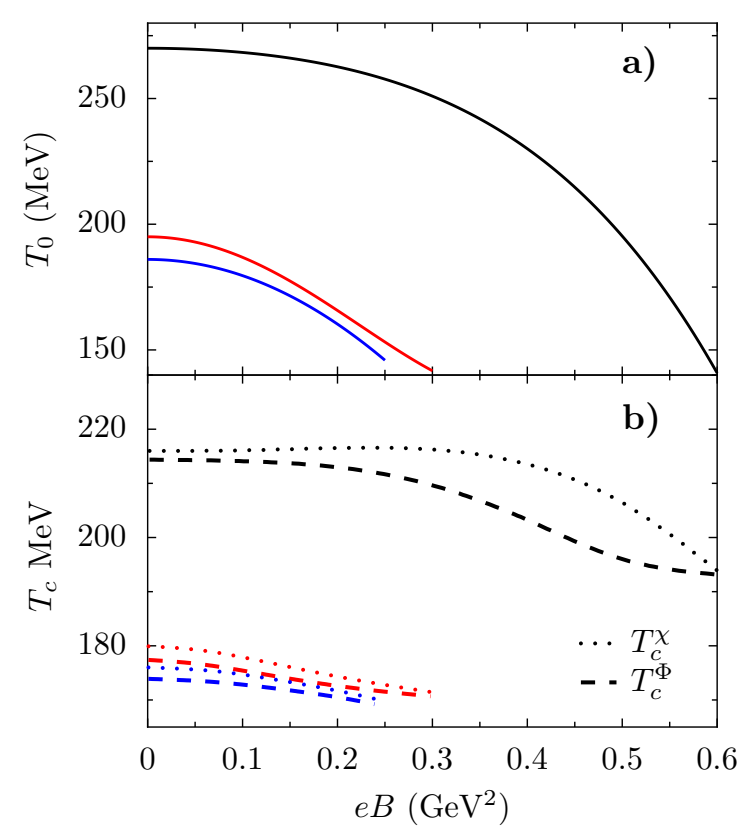

FIG. 7. (a) $T_{0}$ as a function of $e B$ defined by Eq. (9) for the different values of $T_{0}(e B=0)$ presented in Table [V] and (b) the corresponding pseudo-critical temperatures as a function of $e B$ for different values of $T_{0}(e B)$.

is obtained. A similar scenario also occurs if $T_{0}(e B)$ is fitted to reproduce the upper limit of the deconfinement transition shown in Fig. 10 of [11]. At $e B=0$ we have $T_{0}=195 \mathrm{MeV}$ with $T_{c}^{\Phi}=177.4 \mathrm{MeV}$ and $T_{c}^{\chi}=179.9$ $\mathrm{MeV}$. This parametrization is valid for $e B \lesssim 0.3 \mathrm{GeV}^{2}$ (see Table IV] and Fig. [7 red line).

A larger range of validity would have been obtained if the quark back-reaction had not been accounted for (Fig. 7) black lines). In this case we would have for $e B=0, T_{0}=270 \mathrm{MeV}$ as obtained in pure gauge, which gives $T_{c}^{\Phi}=214 \mathrm{MeV}, 40 \mathrm{MeV}$ higher than the prediction of lattice QCD data in [1]. This parametrization also shown in Table IV reproduces lattice QCD data for $T_{c}^{\Phi}(e B)$ [11], shifted by an amount of $40 \mathrm{MeV}$, for magnetic fields up to $0.61 \mathrm{GeV}^{2}$. Above $0.61 \mathrm{GeV}^{2}$, a first order phase transition is obtained. We next use the last scenario to illustrate our results because larger magnetic fields are achieved.

In Fig. 8 (a), $\overline{\Delta \Sigma}$ is plotted as a function of the magnetic field for $e B<0.61 \mathrm{GeV}^{2}$ and several temperatures 

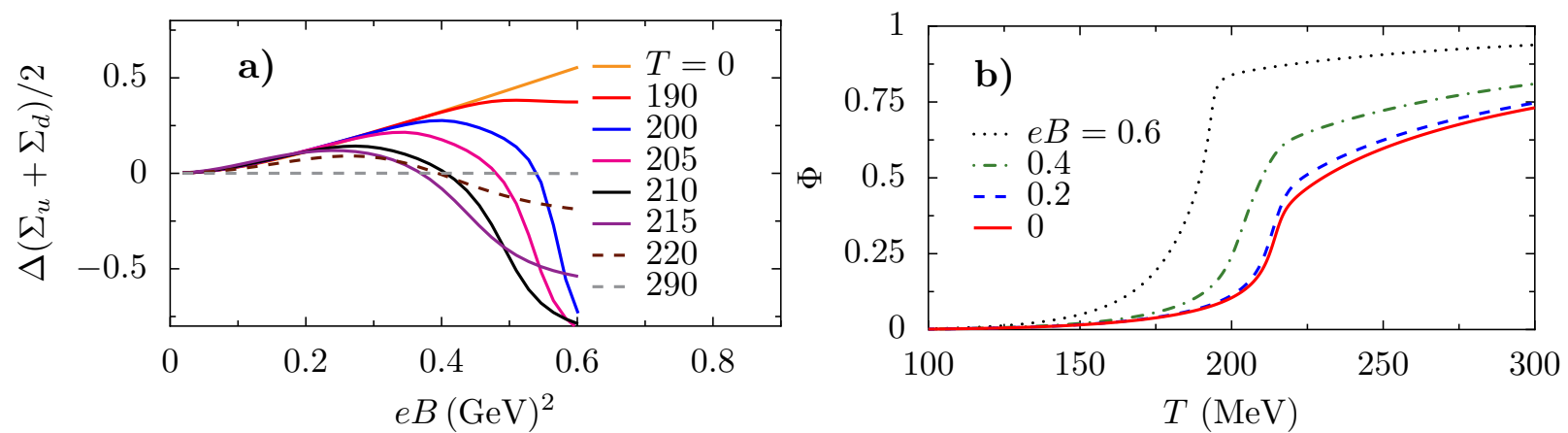

FIG. 8. (a) $\overline{\Delta \Sigma}$ vs $e B \mathrm{GeV}^{2}$ for several temperatures (in $\mathrm{MeV}$ ) within the EPNJL model with $T_{0}(e B)$ defined in Eq. (9) and (b) the Polyakov loop vs $T$ for different values of $e B$ (in $\mathrm{GeV}^{2}$ ).

close to $T_{c}^{\Phi}(e B=0)$. The main conclusions are: (a) the qualitative behavior shown in Fig. 2 of Ref. 221] and in Fig. 6 of Ref. 22 are reproduced, that is, the nonmonotonic behavior of the condensates as a function of the magnetic field; (b) the $T=0$ curve has the highest $\overline{\Delta \Sigma}$, contrary to the results of Fig. 3 (b) for PNJL with fixed $T_{0}$; (c) for $200<T<220 \mathrm{MeV}$ the strong interplay between the partial restoration of chiral symmetry, that for stronger magnetic fields occurs at smaller temperatures (see Table I), gives rise to curves that for small $e B$ values increase (magnetic catalysis) and as soon as the partial restoration of chiral symmetry becomes dominant the curve starts to decrease; (d) for temperatures higher than $190 \mathrm{MeV}$, the curves are negative because $\Delta \Sigma(B, T)$ includes the subtraction of the condensate $\Sigma(0, T)$, when partial restoration of chiral symmetry has not occurred yet; (e) the behavior of the Polyakov loop shown in Fig. 8 (b) follows the same tendency predicted by the lattice calculations 22] and increases with $e B$ for a given $T$. Similar results are obtained if the zero magnetic field Polyakov loop scale $T_{0}$ includes quark back-reaction, however in a smaller range of the magnetic field intensity if we restrict results to a crossover.

To understand the inverse magnetic catalysis phenomenon several studies have been made. The magnetic inhibition can be a feasible explanation for the decreasing behavior of the chiral restoration temperature with increasing $e B$ [40]. Also recently, a mechanism to explain the inverse magnetic catalysis around the critical temperature as induced by sphalerons was proposed [41].

\section{CONCLUSIONS}

In the present work the behavior of the quark condensates at zero chemical potential and finite temperature under the influence of an external magnetic field are studied within three flavor PNJL and EPNJL. The results are compared with the lattice QCD data discussed in [11, 21].

Most of the properties of the quark condensates obtained with the 3 flavor version of PNJL and EPNJL had been obtained with the two flavor versions [13, 25]. In particular, in the present work we have shown that the chiral and deconfinement transition temperatures increase in the presence of an external magnetic field, although the deconfinement transition temperature suffers a much weaker effect. Moreover, it was shown within the SU(3) PNJL and EPNJL models that at $T=0$ the quantitative behavior of light quark condensates with the magnetic field is closer to the lattice results.

Another aspect that should be referred is the effect of the magnetic field on the EPNJL deconfinement and chiral transition temperatures: the existing coincidence at $e B=0$ is destroyed by the magnetic field. Also, the chiral and the deconfinement transition temperatures behave differently with the magnetic field in both models: the deconfinement temperature suffers just a small increase compared to the huge increase of the chiral transition temperature within PNJL, while the increase of the deconfinement phase transition is almost three times as large in the EPNJL due to the entanglement interaction.

The light quark condensates are in good agreement with the LQCD results, in particular, for lower values of $e B$, although, a significative difference between PNJL and lattice calculations occurs for larger values of $e B$. The lattice predicts a larger difference between both condensates at temperatures well below the transition temperature. Close to the transition temperature the lattice predicts a softer restoration of the chiral symmetry. These two features seem to indicate, that compared with PNJL and EPNJL in the lattice calculations, the effect due to the electric charge quark difference is stronger and the restoration of the $u$ quark chiral symmetry starts at lower temperatures. The light quarks susceptibility width does not suffer a large effect with $e B$ just like in LQCD, while the height of the $u$ quark susceptibility slightly increases (PNJL) or even decreases (EPNJL) while in LQCD it suffers a large increase if $e B$ increases from 0 to $1 \mathrm{GeV}^{2}$.

The magnetic field back-reaction on the Polyakov loop may be taken into account using a magnetic field dependent scale parameter $T_{0}$ which reproduces the lattice transition temperatures. When this is done the behavior 
of the Polyakov loop with $e B$ follows the lattice trend. However, within EPNJL a first order phase transition, instead of a crossover, is obtained above $\sim 0.3 \mathrm{GeV}^{2}$ $\left(\sim 0.61 \mathrm{GeV}^{2}\right)$ taking (not taking) into account the quark back-reaction on the Polyakov loop. Understanding the origin of these effects needs further investigation.

Acknowledgments: This work was partially supported by Project No. PTDC/FIS/ 113292/2009 developed under the initiative QREN financed by the
UE/FEDER through the program COMPETE - "Programa Operacional Factores de Competitividade", by Grant No. SFRH/BD/51717/2011, by CNPq/Brazil and FAPESC/Brazil, by CONICET (Argentina) under Grant No. PIP 00682, and by ANPCyT (Argentina) under grant PICT11-03-113. We thank fruitful discussions with Dr. M. B. Pinto and the use of one preliminar code developed by Dr. S. S. Avancini. We are also grateful to F. Bruckmann and G. Endrodi for their lattice data. N. N. S. also would like to thank P. Allen for useful discussions.
[1] R. C. Duncan and C. Thompson, Astrophys. J. 392, L9 (1992); C. Kouveliotou et al., Nature 393, 235 (1998).

[2] V. Skokov, A. Y. Illarionov, and V. Toneev, Int. J. Mod. Phys. A 24, 5925 (2009); V. Voronyuk, V. Toneev, W. Cassing, E. Bratkovskaya, V. Konchakovski, and S. Voloshin, Phys. Rev. C 83, 054911 (2011).

[3] D. E. Kharzeev, L. D. McLerran and H. J. Warringa, Nucl. Phys. A 803, 227 (2008).

[4] T. Vachaspati, Phys. Lett. B 265, 258 (1991); K. Enqvist and P. Olesen, Phys. Lett. B 319, 178 (1993).

[5] K. G. Klimenko, Z. Phys. C 54, 323 (1992).

[6] D. Ebert and K. G. Klimenko, Nucl. Phys. A 728, 203 (2003).

[7] E. J. Ferrer, V. de la Incera and C. Manuel, Phys. Rev. Lett. 95, 152002 (2005).

[8] A. J. Mizher, M. N. Chernodub and E. S. Fraga, Phys. Rev. D 82, 105016 (2010).

[9] B. Chatterjee, H. Mishra and A. Mishra, Phys. Rev. D 84, 014016 (2011).

[10] M. N. Chernodub, Phys. Rev. Lett. 106, 142003 (2011).

[11] G. S. Bali, F. Bruckmann, G. Endrödi, Z. Fodor, S. D. Katz, S. Krieg, A. Schäfer, and K. K. Szabó, J. High Energy Phys. 1202, 044 (2012).

[12] M. D'Elia, Lect. Notes Phys. 871, 181 (2013).

[13] R. Gatto and M. Ruggieri, Lect. Notes Phys. 871, 87 (2013).

[14] E. S. Fraga, Lect. Notes Phys. 871, 121 (2013).

[15] S. P. Klevansky and R. H. Lemmer, Phys. Rev. D 39, 3478 (1989).

[16] Sidney S. Avancini, Débora P. Menezes, Marcus B. Pinto, and Constança Providência, Phys. Rev. D (R) 85, 091901 (2012).

[17] M. D'Elia, S. Mukherjee, and F. Sanfilippo, Phys. Rev. D 82, 051501 (2010).

[18] M. D'Elia and F. Negro, Phys. Rev. D 83, 114028 (2011).

[19] E.-M. Ilgenfritz, M. Kalinowski, M. Müller-Preussker, B. Petersson, and A. Schreiber, Phys. Rev. D 85, 114504 (2012).

[20] E.-M. Ilgenfritz, M. Müller-Preussker, B. Petersson, and A. Schreiber, arXiv:1310.7876 1 [hep-lat].

[21] G. S. Bali, F. Bruckmann, G. Endrödi, Z. Fodor, S. D. Katz, and A. Schäfer, Phys. Rev. D 86, 071502 (2012).

[22] F. Bruckmann, G. Endrodi and T. G. Kovacs, J. High Energy Phys. 1304, 112 (2013).

[23] K. Fukushima, Phys. Lett. B 591, 277 (2004); C. Ratti,
M. A. Thaler, and W. Weise, Phys. Rev. D 73, 014019 (2006); E. Megias, E. Ruiz Arriola and L. L. Salcedo, Phys. Rev. D 74, 065005 (2006).

[24] Y. Sakai, T. Sasaki, H. Kouno, and M. Yahiro, Phys. Rev. D 82, 076003 (2010); J. Phys. G 39, 035004 (2012). T. Sasaki, Y. Sakai, H. Kouno, and M. Yahiro, Phys. Rev. D 84, 091901(R) (2011).

[25] K. Fukushima, M. Ruggieri and R. Gatto, Phys. Rev. D 81, 114031 (2010);

[26] T. Hatsuda and T. Kunihiro, Phys. Rep. 247, 221 (1994); S.P. Klevansky, Rev. Mod. Phys. 64, 649 (1992).

[27] M. Buballa, Phys. Rep. 407, 205 (2005).

[28] S. Roessner, C. Ratti and W. Weise, Phys. Rev. D 75, 034007 (2007).

[29] P. Rehberg, S.P. Klevansky, and J. Hüfner, Phys. Rev. C 53, 410 (1996).

[30] D.P. Menezes, M.B. Pinto, S.S. Avancini, A. Pérez Martínez and C. Providência, Phys. Rev. C 79, 035807 (2009); D.P. Menezes, M.B. Pinto, S.S. Avancini and C. Providência, Phys. Rev. C 80, 065805 (2009).

[31] S.S. Avancini, D.P. Menezes and C. Providência, Phys. Rev. C 83, 065805 (2011).

[32] P. Costa, M.C. Ruivo, C.A. de Sousa and H. Hansen, Symmetry 2(3), 1338 (2010); P. Costa, M. C. Ruivo, C. A. de Sousa, H. Hansen and W. M. Alberico, Phys. Rev. D 79, 116003 (2009).

[33] R. Gatto and M. Ruggieri, Phys. Rev. D 82, 054027 (2010); Phys. Rev. D 83, 034016.

[34] V. Skokov, Phys. Rev. D 85, 034026 (2012).

[35] Seung-il Nam and Chung-Wen Kao, Phys. Rev. D 83, 096009 (2011).

[36] V. A. Miransky and I. A. Shovkovy, Phys. Rev. D 66, 045006 (2002).

[37] T. Kojo and N. Su, Phys. Lett. B 720, 192 (2013).

[38] B. -J. Schaefer, J. M. Pawlowski and J. Wambach, Phys. Rev. D 76 , 074023 (2007); B. -J. Schaefer, M. Wagner and J. Wambach, Phys. Rev. D 81 , 074013 (2010); T. K. Herbst, J. M. Pawlowski and B. J. Schaefer, Phys. Lett. B 696, 58 (2011).

[39] T. K. Herbst, J. M. Pawlowski and B.-J. Schaefer, Phys. Lett. B 696, 58 (2011).

[40] K. Fukushima and Y. Hidaka, Phys. Rev. Lett. 110, 031601 (2013).

[41] J. Chao, P. Chu and M. Huang, Phys. Rev. D 88, 054009 (2013). 